\title{
A second-order accurate scheme for two-dimensional space fractional diffusion equations with time Caputo-Fabrizio fractional derivative
}

\author{
Jiankang Shi · Minghua Chen
}

Received: date / Accepted: date

\begin{abstract}
We provide and analyze a second order scheme for the model describing the functional distributions of particles performing anomalous motion with exponential Debye pattern and no-time-taking jumps eliminated, and power-law jump length. The model is derived in [M. Chen, J. Shi, W. Deng, arXiv:1809.03263], being called the space fractional diffusion equation with the time Caputo-Fabrizio fractional derivative. The designed schemes are unconditionally stable and have the second order global truncation error with the nonzero initial condition, being theoretically proved and numerically verified by two methods (a prior estimate with $L^{2}$-norm and mathematical induction with $l_{\infty}$ norm). Moreover, the optimal estimates are obtained.
\end{abstract}

Keywords Caputo-Fabrizio fractional derivative $\cdot$ Stability and convergence $\cdot$ Optimal estimates

\section{Introduction}

The Caputo-Fabrizio fractional derivative [9] has been used to model a variety of applied scientific phenomena, such as control systems [4], physics [1] 6], medicine [27], fluid dynamics [2, 5, 22, 26]. It is able to describe the material heterogeneities and the fluctuations of different scales. Based on the continuous time random walk with exponential Debye pattern and no-time-taking jumps eliminated, and power-law jump length, i.e., taking the waiting time distribution is $\sigma(1+\sigma-\delta(t)) \exp (-\sigma t), \sigma=\gamma /(1-\gamma)$ and the jump length distribution is $|x|^{-1-\alpha}$, we derive the following space fractional diffusion equation with the time Caputo-Fabrizio fractional operator [13]

$$
\left\{\begin{array}{l}
{ }_{0}^{C F} D_{t}^{\gamma} u(x, y, t)=\frac{\partial^{\alpha} u(x, y, t)}{\partial|x|^{\alpha}}+\frac{\partial^{\beta} u(x, y, t)}{\partial|y|^{\beta}}+f(x, y, t), \\
u(x, y, 0)=u_{0}(x, y) \text { for }(x, y) \in \Omega \\
u(x, y, t)=0 \text { for }(x, y, t) \in \partial \Omega \times[0, T]
\end{array}\right.
$$

J. Shi · M. Chen $(\bowtie)$

School of Mathematics and Statistics, Gansu Key Laboratory of Applied Mathematics and Complex Systems, Lanzhou University, Lanzhou 730000, P.R. China email: shijk17@1zu.edu.cn; chenmh@1zu.edu.cn; 
on a finite rectangular domain $\Omega=\left(0, x_{R}\right) \times\left(0, y_{R}\right)$ and $0<t \leq T$. The Caputo-Fabrizio fractional derivative, for $0<\gamma<1$, is defined by [9] 13$]$

$$
{ }_{0}^{C F} D_{t}^{\gamma} u(t)=\frac{1}{1-\gamma} \int_{0}^{t} u^{\prime}(s) e^{-\frac{\gamma}{1-\gamma}(t-s)} d s=\frac{1}{1-\gamma} \int_{0}^{t} u^{\prime}(s) e^{-\sigma(t-s)} d s, \quad \sigma=\frac{\gamma}{1-\gamma} .
$$

The Riesz fractional derivative is given in [24]

$$
\frac{\partial^{\alpha} u(x, t)}{\partial|x|^{\alpha}}=\kappa_{\alpha}\left({ }_{0} D_{x}^{\alpha}+{ }_{x} D_{x_{R}}^{\alpha}\right) u(x, t), \quad \kappa_{\alpha}=-\frac{1}{2 \cos (\alpha \pi / 2)}>0,1<\alpha<2
$$

with

$$
\begin{aligned}
& { }_{0} D_{x}^{\alpha} u(x, t)=\frac{1}{\Gamma(2-\alpha)} \frac{\partial^{2}}{\partial x^{2}} \int_{0}^{x}(x-\xi)^{1-\alpha} u(\xi, t) d \xi, \\
& { }_{x} D_{x_{R}}^{\alpha} u(x, t)=\frac{1}{\Gamma(2-\alpha)} \frac{\partial^{2}}{\partial x^{2}} \int_{x}^{b}(\xi-x)^{1-\alpha} u(\xi, t) d \xi .
\end{aligned}
$$

In recently years, numerical method for solving the Caputo-Fabrizio fractional derivative [9] is experiencing rapid development. For example, the new operational matrix together with Tau method has been used to solve the equation with Caputo-Fabrizio operator [20]. Numerical approach of Fokker-Planck equation with Caputo-Fabrizio fractional derivative is discussed in [16]. A second-order Crank-Nicolson scheme [28] of the time fractional Caputo-Fabrizio derivative with $1<\gamma<2$ is proposed in [19]; and the stability analysis of the numerical scheme for the groundwater model with Caputo-Fabrizio operator are proven in [15]. Using the Lubich's operator [21] and the discretized fractional substantial calculus [10,11], the stability of the second-order scheme for Caputo-Fabrizio fractional equation are proved [13] by a priori estimate [17] under the zero initial condition. Based on the idea of L1 formula [18, 23], a numerical approximation to the Caputo-Fabrizio derivative by using a linear interpolation is provided [3]; and the first-order convergence analyse are given in [7]. It seems that achieving a second-order accurate scheme for L1 formula is not an easy task. This paper focused on providing effective and a second-order accurate scheme for (1.1). Under the nonzero initial condition, the numerical stability and convergence of the L1 scheme with second-order accuracy are theoretically proved by two ways (a prior estimate with $L^{2}$-norm and mathematical induction with $L_{\infty}$ norm); and the optimal estimates are obtained.

The paper is organized as follows. In Section 2, we provide the approximation scheme to the Caputo-Fabrizio fractional derivative, and the full discretization of 1.1. In Section 3 , the unconditionally stability and convergence of the numerical schemes are proved in detail. In Section 4, we use the numerical example to verify the unconditionally stability and the convergence order of the difference schemes. Finally, we conclude the paper with some remarks.

\section{Discretization schemes}

Let the mesh points $x_{i}=i \Delta x, i=0,1,2, \cdots, N_{x}, y_{j}=j \Delta y, j=0,1,2, \cdots, N_{y}$ and $t_{n}=n \tau$, $n=0,1,2, \cdots, N$, where $\Delta x=\frac{x_{R}}{N_{x}}, \Delta y=\frac{y_{R}}{N_{y}}$ and $\tau=\frac{T}{N}$ are the uniform space stepsize and time steplength, respectively. Denote $u_{i, j}^{n}$ as the numerical approximation to $u\left(x_{i} \cdot y_{j}, t_{n}\right)$ and $f_{i, j}^{n}=f\left(x_{i}, y_{j}, t_{n}\right)$. 
2.1 Discretized Caputo-Fabrizio fractional derivative

In this subsection we provide a second-order discretization L1 formula for the CaputoFabrizio fractional derivative, although there is less than the second-order convergence for the Caputo fractional derivative [18].

Lemma 2.1 Let $0<\gamma<1$ with $\sigma=\frac{\gamma}{1-\gamma}$. Let $u(t)$ be sufficiently smooth for $t \geq 0$. Then

$$
{ }_{0}^{C F} D_{t}^{\gamma} u\left(t_{n}\right)=\frac{1}{1-\gamma} \sum_{k=1}^{n} \frac{u\left(t_{k}\right)-u\left(t_{k-1}\right)}{\sigma \tau} e^{-\sigma(n-k) \tau}\left(1-e^{-\sigma \tau}\right)+\mathscr{O}\left(\tau^{2}\right) .
$$

Proof We can rewrite (1.2) as

$$
\begin{aligned}
{ }_{0}^{C F} D_{t}^{\gamma} u\left(t_{n}\right) & =\frac{1}{1-\gamma} \int_{0}^{t_{n}} u^{\prime}(s) e^{-\sigma\left(t_{n}-s\right)} d s=\frac{1}{1-\gamma} \sum_{k=1}^{n} \int_{t_{k-1}}^{t_{k}} u^{\prime}(s) e^{-\sigma\left(t_{n}-s\right)} d s \\
& =\frac{1}{1-\gamma} \sum_{k=1}^{n} \frac{u\left(t_{k}\right)-u\left(t_{k-1}\right)}{\tau} \int_{t_{k-1}}^{t_{k}} e^{-\sigma\left(t_{n}-s\right)} d s+r_{\tau}^{n} \\
& =\frac{1}{1-\gamma} \sum_{k=1}^{n} \frac{u\left(t_{k}\right)-u\left(t_{k-1}\right)}{\sigma \tau} e^{-\sigma(n-k) \tau}\left(1-e^{-\sigma \tau}\right)+r_{\tau}^{n}
\end{aligned}
$$

Here $r_{\tau}^{n}=-I_{1}-I_{2}$ with

$$
\begin{aligned}
& I_{1}=\frac{1}{1-\gamma} \sum_{k=1}^{n} \int_{t_{k-1}}^{t_{k}} \frac{u^{\prime \prime}(s)\left(t_{k}+t_{k-1}-2 s\right)}{2} e^{-\sigma\left(t_{n}-s\right)} d s \\
& I_{2}=\frac{1}{1-\gamma} \sum_{k=1}^{n} \int_{t_{k-1}}^{t_{k}} \frac{\left(t_{k}-s\right)^{3} u^{\prime \prime \prime}\left(\zeta_{1 k}\right)-\left(t_{k-1}-s\right)^{3} u^{\prime \prime \prime}\left(\zeta_{2 k}\right)}{6 \tau} e^{-\sigma\left(t_{n}-s\right)} d s,
\end{aligned}
$$

and $\zeta_{1 k}, \zeta_{2 k} \in\left(t_{k-1}, t_{k}\right)$

Next we shall estimate $r_{\tau}^{n}=\mathscr{O}\left(\tau^{2}\right)$. According to the first equation of 2.1), the first mean value theorem for definite integrals and Taylor series expansion, we have

$$
\begin{aligned}
I_{1}= & \frac{1}{1-\gamma} \sum_{k=1}^{n} \int_{t_{k-1}}^{t_{k-\frac{1}{2}}} \frac{u^{\prime \prime}(s)\left(t_{k}+t_{k-1}-2 s\right)}{2} e^{-\sigma\left(t_{n}-s\right)} d s \\
& +\frac{1}{1-\gamma} \sum_{k=1}^{n} \int_{t_{k-\frac{1}{2}}}^{t_{k}} \frac{u^{\prime \prime}(s)\left(t_{k}+t_{k-1}-2 s\right)}{2} e^{-\sigma\left(t_{n}-s\right)} d s \\
= & \frac{1}{1-\gamma} \sum_{k=1}^{n} \frac{u^{\prime \prime}\left(\eta_{1 k}\right)}{2} \int_{t_{k-1}}^{t_{k-\frac{1}{2}}}\left(t_{k}+t_{k-1}-2 s\right) e^{-\sigma\left(t_{n}-s\right)} d s \\
& +\frac{1}{1-\gamma} \sum_{k=1}^{n} \frac{u^{\prime \prime}\left(\eta_{2 k}\right)}{2} \int_{t_{k-\frac{1}{2}}}^{t_{k}}\left(t_{k}+t_{k-1}-2 s\right) e^{-\sigma\left(t_{n}-s\right)} d s \\
= & I_{11}+I_{12},
\end{aligned}
$$

where

$$
\begin{aligned}
& I_{11}=\frac{1}{2(1-\gamma)} \sum_{k=1}^{n} u^{\prime \prime}\left(\eta_{1 k}\right) \int_{t_{k-1}}^{t_{k}}\left(t_{k}+t_{k-1}-2 s\right) e^{-\sigma\left(t_{n}-s\right)} d s \\
& I_{12}=\frac{1}{2(1-\gamma)} \sum_{k=1}^{n}\left(\eta_{2 k}-\eta_{1 k}\right) u^{\prime \prime \prime}\left(\eta_{3 k}\right) \int_{t_{k-\frac{1}{2}}}^{t_{k}}\left(t_{k}+t_{k-1}-2 s\right) e^{-\sigma\left(t_{n}-s\right)} d s
\end{aligned}
$$


with $\eta_{1 k}, \eta_{2 k}, \eta_{3 k} \in\left(t_{k-1}, t_{k}\right)$. From the definite integrals of 2.2 , there exists

$$
\begin{aligned}
I_{3 k} & =\int_{t_{k-1}}^{t_{k}}\left(t_{k}+t_{k-1}-2 s\right) e^{-\sigma\left(t_{n}-s\right)} d s \\
& =e^{-\sigma(n-k) \tau}\left[-\frac{\tau}{\sigma}\left(1+e^{-\sigma \tau}\right)+\frac{2}{\sigma^{2}}\left(1-e^{-\sigma \tau}\right)\right]=e^{-\sigma(n-k) \tau}\left[-\frac{\sigma}{6} \tau^{3}+\mathscr{O}\left(\tau^{4}\right)\right]
\end{aligned}
$$

and

$$
\begin{aligned}
I_{4 k} & =\int_{t_{k-\frac{1}{2}}}^{t_{k}}\left(t_{k}+t_{k-1}-2 s\right) e^{-\sigma\left(t_{n}-s\right)} d s \\
& =e^{-\sigma(n-k) \tau}\left[\frac{2}{\sigma^{2}}\left(1-e^{-\frac{1}{2} \sigma \tau}\right)-\frac{\tau}{\sigma}\right]=e^{-\sigma(n-k) \tau}\left[-\frac{1}{4} \tau^{2}+\mathscr{O}\left(\tau^{3}\right)\right] .
\end{aligned}
$$

Therefore, we have

$$
\begin{aligned}
& I_{11}=\frac{1}{2(1-\gamma)} \sum_{k=1}^{n} u^{\prime \prime}\left(\eta_{1 k}\right) I_{3 k}=\mathscr{O}\left(\tau^{2}\right), \\
& I_{12}=\frac{1}{2(1-\gamma)} \sum_{k=1}^{n}\left(\eta_{2 k}-\eta_{1 k}\right) u^{\prime \prime \prime}\left(\eta_{3 k}\right) I_{4 k}=\mathscr{O}\left(\tau^{2}\right),
\end{aligned}
$$

where we use $\eta_{2 k}-\eta_{1 k}=\mathscr{O}(\tau)$. From the above equations, we get

$$
I_{1}=I_{11}+I_{12}=\mathscr{O}\left(\tau^{2}\right) .
$$

Since

$$
I_{2}=I_{21}+I_{22}
$$

with

$$
\begin{aligned}
& I_{21}=\frac{1}{6 \tau(1-\gamma)} \sum_{k=1}^{n} u^{\prime \prime \prime}\left(\zeta_{1 k}\right) \int_{t_{k-1}}^{t_{k}}\left(t_{k}-s\right)^{3} e^{-\sigma\left(t_{n}-s\right)} d s \\
& I_{22}=-\frac{1}{6 \tau(1-\gamma)} \sum_{k=1}^{n} u^{\prime \prime \prime}\left(\zeta_{2 k}\right) \int_{t_{k-1}}^{t_{k}}\left(t_{k-1}-s\right)^{3} e^{-\sigma\left(t_{n}-s\right)} d s .
\end{aligned}
$$

It is easy to check that

$$
\begin{aligned}
I_{5 k} & =\int_{t_{k-1}}^{t_{k}}\left(t_{k}-s\right)^{3} e^{-\sigma\left(t_{n}-s\right)} d s=e^{-\sigma(n-k+1) \tau}\left[\frac{6}{\sigma^{4}}\left(e^{\sigma \tau}-1\right)-\frac{6 \tau}{\sigma^{3}}-\frac{3 \tau^{2}}{\sigma^{2}}-\frac{\tau^{3}}{\sigma}\right] \\
& =e^{-\sigma(n-k+1) \tau}\left[\frac{1}{4} \tau^{4}+\mathscr{O}\left(\tau^{5}\right)\right],
\end{aligned}
$$

and

$$
\begin{aligned}
I_{6 k} & =\int_{t_{k-1}}^{t_{k}}\left(t_{k-1}-s\right)^{3} e^{-\sigma\left(t_{n}-s\right)} d s=e^{-\sigma(n-k) \tau}\left[\frac{6}{\sigma^{4}}\left(1-e^{-\sigma \tau}\right)-\frac{6 \tau}{\sigma^{3}}+\frac{3 \tau^{2}}{\sigma^{2}}-\frac{\tau^{3}}{\sigma}\right] \\
& =e^{-\sigma(n-k) \tau}\left[-\frac{1}{4} \tau^{4}+\mathscr{O}\left(\tau^{5}\right)\right] .
\end{aligned}
$$

It yields

$$
I_{2}=I_{21}+I_{22}=\mathscr{O}\left(\tau^{2}\right) .
$$

According to the above equations, we have

$$
\left|r_{\tau}^{n}\right| \leq\left|I_{1}\right|+\left|I_{2}\right|=\mathscr{O}\left(\tau^{2}\right) .
$$

The proof is completed. 
2.2 Derivation of numerical schemes for 1D

Consider the one-dimensional time-space Caputo-Riesz fractional diffusion equation

$$
{ }_{0}^{C F} D_{t}^{\gamma} u(x, t)=\frac{\partial^{\alpha} u(x, t)}{\partial|x|^{\alpha}}+f(x, t) .
$$

To discrete the Riesz fractional derivative for $1<\alpha<2$, we notice that the approximation operator of [1.3] is given in [14]

$$
\begin{aligned}
& \delta_{\alpha,+x} u_{i}^{n}:=\frac{1}{\Gamma(4-\alpha)(\Delta x)^{\alpha}} \sum_{m=0}^{i+1} g_{m}^{\alpha} u_{i-m+1}^{n}=\frac{1}{\Gamma(4-\alpha)(\Delta x)^{\alpha}} \sum_{m=0}^{i+1} g_{i-m+1}^{\alpha} u_{m}^{n} \\
& \delta_{\alpha,-x} u_{i}^{n}:=\frac{1}{\Gamma(4-\alpha)(\Delta x)^{\alpha}} \sum_{m=0}^{N_{x}-i+1} g_{m}^{\alpha} u_{i+m-1}^{n}=\frac{1}{\Gamma(4-\alpha)(\Delta x)^{\alpha}} \sum_{m=i-1}^{N_{x}} g_{m-i+1}^{\alpha} u_{m}^{n},
\end{aligned}
$$

and there exists

$$
\left.{ }_{0} D_{x}^{\alpha} u(x, t)\right|_{x=x_{i}}=\delta_{\alpha,+x} u_{i}^{n}+\mathscr{O}\left((\Delta x)^{2}\right) \text { and }\left.{ }_{x} D_{x_{R}}^{\alpha} u(x, t)\right|_{x=x_{i}}=\delta_{\alpha,-x} u_{i}^{n}+\mathscr{O}\left((\Delta x)^{2}\right)
$$

with

$$
g_{m}^{\alpha}= \begin{cases}1, & m=0 \\ -4+2^{3-\alpha}, & m=1 \\ 6-2^{5-\alpha}+3^{3-\alpha}, & m=2 \\ (m+1)^{3-\alpha}-4 m^{3-\alpha}+6(m-1)^{3-\alpha}-4(m-2)^{3-\alpha}+(m-3)^{3-\alpha} & m \geq 3\end{cases}
$$

Hence, the discrete scheme of the Riesz fractional derivative is

$$
\begin{aligned}
\left.\frac{\partial^{\alpha} u(x, t)}{\partial|x|^{\alpha}}\right|_{x=x_{i}} & =\left.\kappa_{\alpha}\left({ }_{0} D_{x}^{\alpha}+{ }_{x} D_{x_{R}}^{\alpha}\right) u(x, t)\right|_{x=x_{i}}=\kappa_{\alpha}\left(\delta_{\alpha,+x}+\delta_{\alpha,-x}\right) u_{i}^{n}+\mathscr{O}\left((\Delta x)^{2}\right) \\
& =\frac{\kappa_{\alpha}}{\Gamma(4-\alpha)(\Delta x)^{\alpha}} \sum_{m=0}^{N_{x}} \widetilde{g}_{i, m}^{\alpha} u_{m}^{n}+\mathscr{O}\left((\Delta x)^{2}\right),
\end{aligned}
$$

where

$$
g_{i, m}^{\alpha}= \begin{cases}g_{i-m+1}^{\alpha}, & m<i-1 \\ g_{0}^{\alpha}+g_{2}^{\alpha}, & m=i-1 \\ 2 g_{1}^{\alpha}, & m=i \\ g_{0}^{\alpha}+g_{2}^{\alpha}, & m=i+1 \\ g_{m-i+1}^{\alpha}, & m>i+1\end{cases}
$$

with $i=1,2, \cdots, N_{x}-1, u_{0}^{n}$ and $u_{N_{x}}^{n}$ are the boundary conditions.

Taking $u=\left[u\left(x_{1}\right), u\left(x_{2}\right), \cdots, u\left(x_{N_{x}-1}\right)\right]^{T}$, then

$$
\left[\sum_{m=0}^{N_{x}} g_{1, m}^{\alpha} u\left(x_{m}\right), \sum_{m=0}^{N_{x}} g_{2, m}^{\alpha} u\left(x_{m}\right), \cdots, \sum_{m=0}^{N_{x}} g_{N_{x}-1, m}^{\alpha} u\left(x_{m}\right)\right]=A_{\alpha} u
$$

where

$$
A_{\alpha}=B_{\alpha}+B_{\alpha}^{T} \quad \text { with } \quad B_{\alpha}=\left[\begin{array}{cccccc}
g_{1}^{\alpha} & g_{2}^{\alpha} & g_{3}^{\alpha} & \cdots & g_{N_{x}-2}^{\alpha} & g_{N_{x}-1}^{\alpha} \\
g_{0}^{\alpha} & g_{1}^{\alpha} & g_{2}^{\alpha} & \cdots & g_{N_{x}-3}^{\alpha} & g_{N_{x}-2}^{\alpha} \\
0 & g_{0}^{\alpha} & g_{1}^{\alpha} & \cdots & g_{N_{x}-4}^{\alpha} & g_{N_{x}-3}^{\alpha} \\
\vdots & \vdots & \vdots & \ddots & \vdots & \vdots \\
0 & 0 & 0 & \cdots & g_{1}^{\alpha} & g_{2}^{\alpha} \\
0 & 0 & 0 & \cdots & g_{0}^{\alpha} & g_{1}^{\alpha}
\end{array}\right]_{\left(N_{x}-1\right) \times\left(N_{x}-1\right)}
$$


According to (2.5) and Lemma 2.1, we can rewrite 2.3) as

$$
\frac{1}{1-\gamma} \sum_{k=1}^{n} \frac{u_{i}^{k}-u_{i}^{k-1}}{\sigma \tau} e^{-\sigma(n-k) \tau}\left(1-e^{-\sigma \tau}\right)=\frac{\kappa_{\alpha}}{\Gamma(4-\alpha)(\Delta x)^{\alpha}} \sum_{m=0}^{N_{x}} g_{i, m}^{\alpha} u_{m}^{n}+f_{i}^{n}+r_{i}^{n}
$$

with the local truncation error

$$
\left|r_{i}^{n}\right| \leq C_{u}\left(\tau^{2}+(\Delta x)^{2}\right),
$$

where the positive constant $C_{u}$ independent of $\tau$ and $h$.

Therefore the resulting discretization of (2.3) is

$$
\frac{1}{1-\gamma} \sum_{k=1}^{n} \frac{u_{i}^{k}-u_{i}^{k-1}}{\sigma \tau} e^{-\sigma(n-k) \tau}\left(1-e^{-\sigma \tau}\right)=\frac{\kappa_{\alpha}}{\Gamma(4-\alpha)(\Delta x)^{\alpha}} \sum_{m=0}^{N_{x}} g_{i, m}^{\alpha} u_{m}^{n}+f_{i}^{n},
$$

i.e.,

$$
\begin{aligned}
& \frac{1}{(1-\gamma) \sigma \tau}\left[u_{i}^{n}\left(1-e^{-\sigma \tau}\right)-\sum_{k=1}^{n-1} u_{i}^{k} e^{-\sigma(n-1-k) \tau}\left(1-e^{-\sigma \tau}\right)^{2}\right] \\
& =\frac{\kappa_{\alpha}}{\Gamma(4-\alpha)(\Delta x)^{\alpha}} \sum_{m=0}^{N_{x}} g_{i, m}^{\alpha} u_{m}^{n}+f_{i}^{n}+\frac{1}{(1-\gamma) \sigma \tau} u_{i}^{0}\left(1-e^{-\sigma \tau}\right) e^{-\sigma(n-1) \tau},
\end{aligned}
$$

which is equivalent to

$$
u_{i}^{n}-\kappa_{\Delta x, \tau} \sum_{m=0}^{N_{x}} g_{i, m}^{\alpha} u_{m}^{n}=\sum_{k=1}^{n-1} u_{i}^{k} e^{-\sigma(n-1-k) \tau}\left(1-e^{-\sigma \tau}\right)+u_{i}^{0} e^{-\sigma(n-1) \tau}+\frac{(1-\gamma) \sigma \tau}{1-e^{-\sigma \tau}} f_{i}^{n}
$$

with $\kappa_{\Delta x, \tau}=\frac{(1-\gamma) \sigma \tau}{\left(1-e^{-\sigma \tau}\right)} \frac{\kappa_{\alpha}}{\Gamma(4-\alpha)(\Delta x)^{\alpha}}$.

2.3 Derivation of numerical schemes for $2 \mathrm{D}$

In the same way, we can rewrite 1.1 as

$$
\begin{aligned}
& \frac{1}{1-\gamma} \sum_{k=1}^{n} \frac{u_{i, j}^{k}-u_{i, j}^{k-1}}{\sigma \tau} e^{-\sigma(n-k) \tau}\left(1-e^{-\sigma \tau}\right) \\
& =\frac{\kappa_{\alpha}}{\Gamma(4-\alpha)(\Delta x)^{\alpha}} \sum_{m=0}^{N_{x}} g_{i, m}^{\alpha} u_{m, j}^{n}+\frac{\kappa_{\beta}}{\Gamma(4-\beta)(\Delta y)^{\beta}} \sum_{m=0}^{N_{y}} g_{j, m}^{\beta} u_{i, m}^{n}+f_{i, j}^{n}+r_{i, j}^{n}
\end{aligned}
$$

with the local truncation error

$$
\left|r_{i, j}^{n}\right| \leq C_{u}\left(\tau^{2}+(\Delta x)^{2}+(\Delta y)^{2}\right) .
$$

Therefore the resulting discretization of (1.1) is

$$
\begin{aligned}
& \frac{1}{1-\gamma} \sum_{k=1}^{n} \frac{u_{i, j}^{k}-u_{i, j}^{k-1}}{\sigma \tau} e^{-\sigma(n-k) \tau}\left(1-e^{-\sigma \tau}\right) \\
& =\frac{\kappa_{\alpha}}{\Gamma(4-\alpha)(\Delta x)^{\alpha}} \sum_{m=0}^{N_{x}} g_{i, m}^{\alpha} u_{m, j}^{n}+\frac{\kappa_{\beta}}{\Gamma(4-\beta)(\Delta y)^{\beta}} \sum_{m=0}^{N_{y}} g_{j, m}^{\beta} u_{i, m}^{n}+f_{i, j}^{n},
\end{aligned}
$$


i.e.,

$$
\begin{aligned}
& u_{i, j}^{n}-\kappa_{\Delta x, \tau}^{\alpha} \sum_{m=0}^{N_{x}} g_{i, m}^{\alpha} u_{m, j}^{n}-\kappa_{\Delta y, \tau}^{\alpha} \sum_{m=0}^{N_{y}} g_{j, m}^{\alpha} u_{i, m}^{n} \\
& =\sum_{k=1}^{n-1} u_{i, j}^{k} e^{-\sigma(n-1-k) \tau}\left(1-e^{-\sigma \tau}\right)+u_{i, j}^{0} e^{-\sigma(n-1) \tau}+\frac{(1-\gamma) \sigma \tau}{1-e^{-\sigma \tau}} f_{i, j}^{n}
\end{aligned}
$$

with $\kappa_{\Delta x, \tau}^{\alpha}=\frac{(1-\gamma) \sigma \tau}{\left(1-e^{-\sigma \tau}\right)} \frac{\kappa_{\alpha}}{\Gamma(4-\alpha)(\Delta x)^{\alpha}}$ and $\kappa_{\Delta y, \tau}^{\beta}=\frac{(1-\gamma) \sigma \tau}{\left(1-e^{-\sigma \tau}\right)} \frac{\kappa_{\beta}}{\Gamma(4-\beta)(\Delta y)^{\beta}}$.

\section{Stability and convergence}

In this section, we theoretically prove that the above numerical schemes are unconditionally stable with the nonzero initial conditions. First, we denote $u^{n}=\left\{u_{i}^{n} \mid 0 \leq i \leq N_{x}, n \geq 0\right\}$ and $v^{n}=\left\{v_{i}^{n} \mid 0 \leq i \leq N_{x}, n \geq 0\right\}$, which are grid functions. And we introduce the discrete inner products as following

$$
\left(u^{n}, v^{n}\right)=\Delta x \sum_{i=0}^{N_{x}-1} u_{i}^{n} v_{i}^{n}, \quad\left\|u^{n}\right\|=\left(u^{n}, u^{n}\right)^{1 / 2} .
$$

3.1 A few technical lemmas

Lemma 3.1 [25] p. 27] A matrix $A \in R$ is positive definite in $R$ if $(A x, x)>0, \forall x \in R^{n}, x \neq 0$. A real matrix $A$ of order $n$ is positive definite iff its symmetric part $H=\frac{A+A^{T}}{2}$ is positive definite.

Lemma 3.2 [25] p. 29] A matrix $A \in R^{n \times n}$ is called diagonally dominant by rows if $\left|a_{i i}\right| \geq$ $\sum_{j=1, j \neq i}^{n}\left|a_{i j}\right|$ with $i=1,2, \cdots n$. A strictly diagonally dominant matrix that is symmetric with positive diagonal entries is also positive definite.

Lemma 3.3 ([14]) The coefficients $\widetilde{g}_{i, m}^{\alpha}, \alpha \in(1,2)$ defined in (2.6) satisfy

(1) $g_{i, i}^{\alpha}<0, \quad g_{i, m}^{\alpha}>0 \quad(m \neq i)$;

(2) $\sum_{m=0}^{N_{x}} g_{i, m}^{\alpha}<0$ and $-g_{i, i}^{\alpha}>\sum_{m=0, m \neq i}^{N_{x}} g_{i, m}^{\alpha}$;

(3) $g_{0}^{\alpha}>0, g_{1}^{\alpha}<0, g_{0}^{\alpha}+g_{2}^{\alpha}>0, g_{k}^{\alpha}>0 \quad \forall k \geq 3$.

Lemma 3.4 Let $1<\alpha<2$ and $g_{m}^{\alpha}$ given by (2.4). Then

$$
\sum_{m=0}^{i+1} g_{m}^{\alpha} \leq \frac{1}{i^{\alpha} \Gamma(1-\alpha)}<0
$$


Proof Using (2.13) and (2.4) with $u(x, t)=1$ of [12], we obtain

$$
\begin{aligned}
& \sum_{m=0}^{i+1} g_{m} \\
& =\frac{\Gamma(4-\alpha)(\Delta x)^{\alpha}}{(\Delta x)^{2} \Gamma(2-\alpha)}\left[\int_{0}^{x_{i-1}}\left(x_{i-1}-\xi\right)^{1-\alpha} d \xi-2 \int_{0}^{x_{i}}\left(x_{i}-\xi\right)^{1-\alpha} d \xi+\int_{0}^{x_{i+1}}\left(x_{i+1}-\xi\right)^{1-\alpha} d \xi\right] \\
& =(3-\alpha) i^{2-\alpha}\left[\left(1-\frac{1}{i}\right)^{2-\alpha}-2+\left(1+\frac{1}{i}\right)^{2-\alpha}\right] \\
& \leq \frac{(3-\alpha)(2-\alpha)(1-\alpha)}{i^{\alpha}} \leq \frac{1}{i^{\alpha} \Gamma(1-\alpha)}<0 .
\end{aligned}
$$

The proof is completed.

Lemma 3.5 Let $1<\alpha<2$ and $A_{\alpha}$ be given in (2.7). Then

$$
-\frac{1}{(\Delta x)^{\alpha}}\left(A_{\alpha} v, v\right) \geq-\frac{2}{\Gamma(1-\alpha)\left(x_{R}\right)^{\alpha}}\|v\|^{2}>0 \text { with } v \in \mathbb{R}^{M-1}, \Omega=\left(0, x_{R}\right) .
$$

Proof Let the vector $v=\left(v_{1}, v_{2}, \ldots, v_{N_{x}-1}\right)^{T}$ with $v_{0}=v_{N_{x}}=0$. From 2.7) and Lemma 3.3, there exists

$$
\begin{aligned}
\left(B_{\alpha} v, v\right) & =\Delta x \sum_{i=1}^{N_{x}-1}\left(\sum_{k=0}^{N_{x}-i} g_{k}^{\alpha} v_{i+k-1}\right) v_{i}=\Delta x \sum_{k=0}^{N_{x}-1} g_{k}^{\alpha}\left(\sum_{i=1}^{N_{x}-k} v_{i+k-1} v_{i}\right) \\
& =g_{1}^{\alpha} \Delta x \sum_{i=1}^{N_{x}-1} v_{i}^{2}+\left(g_{0}^{\alpha}+g_{2}^{\alpha}\right) \Delta x \sum_{i=1}^{N_{x}-2} v_{i} v_{i+1}+\Delta x \sum_{k=3}^{N_{x}-1} g_{k}^{\alpha}\left(\sum_{i=1}^{N_{x}-k} v_{i+k-1} v_{i}\right) \\
& \leq g_{1}^{\alpha}\|v\|^{2}+\left(g_{0}^{\alpha}+g_{2}^{\alpha}\right) \Delta x \sum_{i=1}^{N_{x}-2} \frac{v_{i}^{2}+v_{i+1}^{2}}{2}+\Delta x \sum_{k=3}^{N_{x}-1} g_{k}^{\alpha}\left(\sum_{i=1}^{N_{x}-k} \frac{v_{i}^{2}+v_{i+k-1}^{2}}{2}\right) \\
& \leq\left(\sum_{k=0}^{M-1} g_{k}^{\alpha}\right)\|v\|^{2} \leq\left(\sum_{k=0}^{N_{x}+1} g_{k}^{\alpha}\right)\|v\|^{2} .
\end{aligned}
$$

Since $\left(B_{\alpha}^{T} v, v\right)=\left(B_{\alpha} v, v\right)$ and $A_{\alpha}=B_{\alpha}^{T}+B_{\alpha}$, we have

$$
\left(A_{\alpha} v, v\right) \leq 2\left(\sum_{k=0}^{N_{x}+1} g_{k}^{\alpha}\right)\|v\|^{2}<0 .
$$

Using the above inequality and Lemma 3.4 we obtain

$$
\begin{aligned}
-\frac{1}{(\Delta x)^{\alpha}}\left(A_{\alpha} v, v\right) & \geq-\frac{2}{(\Delta x)^{\alpha}}\left(\sum_{k=0}^{N_{x}+1} g_{k}^{\alpha}\right)\|v\|^{2} \geq \frac{2}{(\Delta x)^{\alpha}} \frac{-1}{\left(N_{x}\right)^{\alpha} \Gamma(1-\alpha)}\|v\|^{2} \\
& \geq-\frac{2}{\left(x_{R}\right)^{\alpha} \Gamma(1-\alpha)}\|v\|^{2}>0 \text { with } \Omega=\left(0, x_{R}\right), \quad v \in \mathbb{R}^{N_{x}-1} .
\end{aligned}
$$

The proof is completed.

Lemma 3.6 Let $0<\gamma<1$ and $\sigma=\frac{\gamma}{1-\gamma}$. Then for any vector $V_{i}=\left(v_{i}^{1}, v_{i}^{2}, \cdots, v_{i}^{N}\right) \in R^{N}$, we have

$$
\sum_{n=1}^{N}\left(v_{i}^{n}\left(1-e^{-\sigma \tau}\right)-\sum_{k=1}^{n-1} v_{i}^{k} e^{-\sigma(n-1-k) \tau}\left(1-e^{-\sigma \tau}\right)^{2}\right) v_{i}^{n} \geq 0
$$


Proof By the mathematical induction method, we have

$$
\sum_{n=1}^{N}\left(v_{i}^{n}\left(1-e^{-\sigma \tau}\right)-\sum_{k=1}^{n-1} v_{i}^{k} e^{-\sigma(n-1-k) \tau}\left(1-e^{-\sigma \tau}\right)^{2}\right) v_{i}^{n}=\left(A V_{i}, V_{i}\right),
$$

where

$$
A=\left[\begin{array}{cccccc}
b & 0 & 0 & \cdots & 0 & 0 \\
a_{1} & b & 0 & \cdots & 0 & 0 \\
a_{2} & a_{1} & b & \cdots & 0 & 0 \\
\vdots & \vdots & \vdots & \ddots & \vdots & \vdots \\
a_{N-2} & a_{N-3} & a_{N-4} & \cdots & b & 0 \\
a_{N-1} & a_{N-2} & a_{N-3} & \cdots & a_{1} & b
\end{array}\right]_{N \times N}
$$

with $b=1-e^{-\sigma \tau}$ and $a_{l}=-e^{-\sigma(l-1) \tau}\left(1-e^{-\sigma \tau}\right)^{2}<0, l=1,2, \cdots, N-1$. We next prove the matrix $A$ is positive definite. Since

$$
H=\frac{A+A^{T}}{2}=\left[\begin{array}{cccccc}
b & \frac{a_{1}}{2} & \frac{a_{2}}{2} & \cdots & \frac{a_{N-2}}{a_{2}} & \frac{a_{N-1}}{a_{2-2}} \\
\frac{a_{1}}{2} & b & \frac{a_{1}}{2} & \cdots & \frac{a_{N-3}^{2}}{2} & \frac{a_{N-3}}{2} \\
\frac{a_{2}}{2} & \frac{a_{1}}{2} & b & \cdots & \frac{a_{N-4}}{2} & \frac{a_{N-3}}{2} \\
\vdots & \vdots & \vdots & \ddots & \vdots & \vdots \\
\frac{a_{N-2}}{2} & \frac{a_{N-3}}{2} & \frac{a_{N-4}}{2} & \cdots & b & \frac{a_{1}}{2} \\
\frac{a_{N-1}}{2} & \frac{a_{N-2}}{2} & \frac{a_{N-3}}{2} & \cdots & \frac{a_{1}}{2} & b
\end{array}\right]_{N \times N}
$$

and

$$
\begin{aligned}
\sum_{j=1, j \neq i}^{N}\left|h_{i, j}\right| & \leq 2 \sum_{l=1}^{N-1}\left|\frac{a_{l}}{2}\right|=-\sum_{l=1}^{N-1} a_{l}=\sum_{l=1}^{N-1} e^{-\sigma(l-1) \tau}\left(1-e^{-\sigma \tau}\right)^{2} \\
& =\left(1-e^{-\sigma \tau}\right)\left(1-e^{-\sigma(N-1) \tau}\right)<1-e^{-\sigma \tau}=b,
\end{aligned}
$$

it yields the matrix $H$ is strictly diagonally dominant. Form Lemmas 3.1 and 3.2 we know that the matrix $A$ is positive definite. The proof is completed.

For simplifying the proof of the stability and convergence, we first provide the following a priori estimate.

Lemma 3.7 Suppose $v_{i}^{n}$ is the solution of the difference scheme [2.11), i.e.,

$$
\begin{aligned}
& \frac{1}{(1-\gamma) \sigma \tau}\left[v_{i}^{n}\left(1-e^{-\sigma \tau}\right)-\sum_{k=1}^{n-1} v_{i}^{k} e^{-\sigma(n-1-k) \tau}\left(1-e^{-\sigma \tau}\right)^{2}\right] \\
& \quad=\frac{\kappa_{\alpha}}{\Gamma(4-\alpha)(\Delta x)^{\alpha}} \sum_{m=0}^{N_{x}} g_{i, m}^{\alpha} v_{m}^{n}+f_{i}^{n}+\frac{1}{(1-\gamma) \sigma \tau} v_{i}^{0}\left(1-e^{-\sigma \tau}\right) e^{-\sigma(n-1) \tau}, \\
& v_{i}^{0}=\phi_{i}, \quad 1 \leq i \leq N_{x}-1, \\
& v_{0}^{n}=v_{M}^{n}=0, \quad 0 \leq n \leq N .
\end{aligned}
$$

Then for any positive integer $N$ with $N \tau \leq T$, we have

$$
\begin{aligned}
& \tau \sum_{n=1}^{N}\left\|v^{n}\right\|^{2} \\
& \leq \frac{\left(\Gamma(1-\alpha) \Gamma(4-\alpha)\left(x_{R}\right)^{\alpha}\right)^{2}}{2 \kappa_{\alpha}^{2}} \cdot \tau \sum_{n=1}^{N}\left\|f^{n}\right\|^{2}+\frac{\left(\Gamma(1-\alpha) \Gamma(4-\alpha)\left(x_{R}\right)^{\alpha}\right)^{2} T}{(1-\gamma)^{2} \kappa_{\alpha}^{2}}\left\|v^{0}\right\|^{2} .
\end{aligned}
$$


Proof Multiplying (3.3) by $(\Delta x) v_{i}^{n+1}$ and summing up for $i$ from 1 to $N_{x}-1$, we get

$$
\begin{aligned}
& \frac{1}{(1-\gamma) \sigma \tau} \sum_{i=1}^{N_{x}-1}\left[v_{i}^{n}\left(1-e^{-\sigma \tau}\right)-\sum_{k=1}^{n-1} v_{i}^{k} e^{-\sigma(n-1-k) \tau}\left(1-e^{-\sigma \tau}\right)^{2}\right] h v_{i}^{n} \\
& =\sum_{i=1}^{N_{x}-1}\left[\frac{\kappa_{\alpha}}{\Gamma(4-\alpha)(\Delta x)^{\alpha}} \sum_{m=0}^{N_{x}} g_{i, m}^{\alpha} v_{m}^{n}+f_{i}^{n}+\frac{1}{(1-\gamma) \sigma \tau} v_{i}^{0}\left(1-e^{-\sigma \tau}\right) e^{-\sigma(n-1) \tau}\right](\Delta x) v_{i}^{n} \\
& =\frac{\kappa_{\alpha}}{\Gamma(4-\alpha)(\Delta x)^{\alpha}}\left(A_{\alpha} v^{n}, v^{n}\right)+\left(f^{n}, v^{n}\right)+\frac{1}{(1-\gamma) \sigma \tau}\left(1-e^{-\sigma \tau}\right) e^{-\sigma(n-1) \tau}\left(v^{0}, v^{n}\right) .
\end{aligned}
$$

Multiplying the above equation by $\tau$ and summing up for $n$ from 1 to $N$, we obtain

$$
\begin{aligned}
& \frac{\tau}{(1-\gamma) \sigma \tau} \sum_{i=1}^{N_{x}-1} \sum_{n=1}^{N}\left[v_{i}^{n}\left(1-e^{-\sigma \tau}\right)-\sum_{k=1}^{n-1} v_{i}^{k} e^{-\sigma(n-1-k) \tau}\left(1-e^{-\sigma \tau}\right)^{2}\right](\Delta x) v_{i}^{n} \\
& =\tau \sum_{n=1}^{N} \frac{\kappa_{\alpha}}{\Gamma(4-\alpha)(\Delta x)^{\alpha}}\left(A_{\alpha} v^{n}, v^{n}\right)+\tau \sum_{n=1}^{N}\left(f^{n}, v^{n}\right)+\frac{\tau\left(1-e^{-\sigma \tau}\right)}{(1-\gamma) \sigma \tau} \sum_{n=1}^{N} e^{-\sigma(n-1) \tau}\left(v^{0}, v^{n}\right) .
\end{aligned}
$$

From Lemma 3.5 and Lemma 3.6 we have

$$
\begin{aligned}
& -\frac{2}{\Gamma(1-\alpha)} \frac{\kappa_{\alpha}}{\Gamma(4-\alpha)\left(x_{R}\right)^{\alpha}} \tau \sum_{n=1}^{N}\left\|v^{n}\right\|^{2} \leq-\tau \sum_{n=1}^{N} \frac{\kappa_{\alpha}}{\Gamma(4-\alpha)(\Delta x)^{\alpha}}\left(A_{\alpha} v^{n}, v^{n}\right) \\
& \leq \tau \sum_{n=1}^{N}\left(f^{n}, v^{n}\right)+\frac{1}{(1-\gamma) \sigma \tau}\left(1-e^{-\sigma \tau}\right) \tau \sum_{n=1}^{N} e^{-\sigma(n-1) \tau}\left(v^{0}, v^{n}\right) \\
& \leq \tau \sum_{n=1}^{N}\left\|f^{n}\right\| \cdot\left\|v^{n}\right\|+\frac{1}{(1-\gamma)} \tau \sum_{n=1}^{N}\left\|v^{0}\right\| \cdot\left\|v^{n}\right\| \\
& \leq \tau \sum_{n=1}^{N}\left(\varepsilon\left\|v^{n}\right\|^{2}+\frac{\left\|f^{n}\right\|^{2}}{4 \varepsilon}\right)+\frac{1}{(1-\gamma)} \tau \sum_{n=1}^{N}\left(\eta\left\|v^{n}\right\|^{2}+\frac{\left\|v^{0}\right\|^{2}}{4 \eta}\right),
\end{aligned}
$$

where $\varepsilon, \eta>0$ and $\left(f^{n}, v^{n}\right) \leq\left\|f^{n}\right\| \cdot\left\|v^{n}\right\|$.

Taking $\varepsilon=-\frac{1}{\Gamma(1-\alpha)} \frac{\kappa_{\alpha}}{\Gamma(4-\alpha)\left(x_{R}\right)^{\alpha}}$ and $\eta=-\frac{1-\gamma}{2 \Gamma(1-\alpha)} \frac{\kappa_{\alpha}}{\Gamma(4-\alpha)\left(x_{R}\right)^{\alpha}}$, and using the above inequality, there exists

$$
-\frac{1}{2 \Gamma(1-\alpha)} \frac{\kappa_{\alpha}}{\Gamma(4-\alpha)\left(x_{R}\right)^{\alpha}} \tau \sum_{n=1}^{N}\left\|v^{n}\right\|^{2} \leq \frac{1}{4 \varepsilon} \tau \sum_{n=1}^{N}\left\|f^{n}\right\|^{2}+\frac{1}{(1-\gamma) 4 \eta} \tau \sum_{n=1}^{N}\left\|v^{0}\right\|^{2},
$$

and

$$
\begin{aligned}
\tau \sum_{n=1}^{N}\left\|v^{n}\right\|^{2} & \leq \frac{1-\gamma}{4 \varepsilon \eta} \cdot \tau \sum_{n=1}^{N}\left\|f^{n}\right\|^{2}+\frac{T}{4 \eta^{2}}\left\|v^{0}\right\|^{2} \\
& =\frac{\left(\Gamma(1-\alpha) \Gamma(4-\alpha)\left(x_{R}\right)^{\alpha}\right)^{2}}{2 \kappa_{\alpha}^{2}} \tau \sum_{n=1}^{N}\left\|f^{n}\right\|^{2}+\frac{\left(\Gamma(1-\alpha) \Gamma(4-\alpha)\left(x_{R}\right)^{\alpha}\right)^{2} T}{(1-\gamma)^{2} \kappa_{\alpha}^{2}}\left\|v^{0}\right\|^{2}
\end{aligned}
$$

The proof is completed. 
3.2 Convergence and stability for 1D

In this subsection, we prove that the scheme 2.11) is unconditionally stable and convergence by two methods, i.e., a prior estimate and mathematical induction which correspond to the discrete $L^{2}$-norm and $L_{\infty}$ norm.

Theorem 3.1 The difference scheme (2.11) is unconditionally stable.

Proof From Lemma 3.7 the desired results is obtained.

Theorem 3.2 Let $u_{i}^{n}$ be the approximate solution of $u\left(x_{i}, t_{n}\right)$ computed by the difference scheme 2.11). Let $\varepsilon_{i}^{n}=u\left(x_{i}, t_{n}\right)-u_{i}^{n}$. Then

$$
\tau \sum_{n=1}^{N}\left\|\varepsilon^{n}\right\| \leq \frac{|\Gamma(1-\alpha)| \Gamma(4-\alpha)\left(x_{R}\right)^{\alpha+1 / 2} T}{\sqrt{2} \kappa_{\alpha}} C_{u} \cdot\left(\tau^{2}+(\Delta x)^{2}\right),
$$

where $C_{u}$ is defined by (2.9) and $\left(x_{i}, t_{n}\right) \in\left(0, x_{R}\right) \times(0, T]$ with $N \tau \leq T$.

Proof Let $u\left(x_{i}, t_{n}\right)$ be the exact solution of 2.3) at the mesh point $\left(x_{i}, t_{n}\right)$, and $\varepsilon_{i}^{n}=u\left(x_{i}, t_{n}\right)-$ $u_{i}^{n}$. Subtracting 2.8 from 2.11 with $\varepsilon_{i}^{0}=0$, we obtain

$$
\begin{aligned}
& \frac{1}{(1-\gamma) \sigma \tau}\left[\varepsilon_{i}^{n}\left(1-e^{-\sigma \tau}\right)-\sum_{k=1}^{n-1} \varepsilon_{i}^{k} e^{-\sigma(n-1-k) \tau}\left(1-e^{-\sigma \tau}\right)^{2}\right] \\
& =\frac{\kappa_{\alpha}}{\Gamma(4-\alpha)(\Delta x)^{\alpha}} \sum_{m=0}^{N_{x}} g_{i, m}^{\alpha} \varepsilon_{m}^{n}+r_{i}^{n} .
\end{aligned}
$$

From Lemma 3.7 and 2.97, it holds

$$
\begin{aligned}
\tau \sum_{n=1}^{N}\left\|\varepsilon^{n}\right\|^{2} & \leq \frac{\left(\Gamma(1-\alpha) \Gamma(4-\alpha)\left(x_{R}\right)^{\alpha}\right)^{2}}{2 \kappa_{\alpha}^{2}} \cdot \tau \sum_{n=1}^{N}\left\|r^{n}\right\|^{2} \\
& \leq \frac{\left(\Gamma(1-\alpha) \Gamma(4-\alpha)\left(x_{R}\right)^{\alpha}\right)^{2}}{2 \kappa_{\alpha}^{2}} x_{R} T C_{u}^{2} \cdot\left(\tau^{2}+(\Delta x)^{2}\right)^{2} .
\end{aligned}
$$

Using Cauchy-Schwarz inequality for the above inequality, we have

$$
\begin{aligned}
\left(\tau \sum_{n=1}^{N}\left\|\varepsilon^{n}\right\|\right)^{2} & \leq\left(\tau \sum_{n=1}^{N} 1\right)\left(\tau \sum_{n=1}^{N}\left\|\varepsilon^{n}\right\|^{2}\right) \\
& \leq \frac{\left(\Gamma(1-\alpha) \Gamma(4-\alpha)\left(x_{R}\right)^{\alpha}\right)^{2}}{2 \kappa_{\alpha}^{2}} x_{R} T^{2} C_{u}^{2} \cdot\left(\tau^{2}+(\Delta x)^{2}\right)^{2}
\end{aligned}
$$

and

$$
\tau \sum_{n=1}^{N}\left\|\varepsilon^{n}\right\| \leq \frac{|\Gamma(1-\alpha)| \Gamma(4-\alpha)\left(x_{R}\right)^{\alpha+1 / 2} T}{\sqrt{2} \kappa_{\alpha}} C_{u} \cdot\left(\tau^{2}+(\Delta x)^{2}\right) .
$$

The proof is completed.

Besides the discrete $L^{2}$-norm, the stability and convergence can also be obtained in $L_{\infty}$ norm by the following mathematical induction.

Theorem 3.3 The difference scheme (2.12) is unconditionally stable. 
Proof Let $\widetilde{u}_{i}^{n}$ be the approximate solution of $u_{i}^{n}$, which is the exact solution of the difference scheme 2.12. Denoting $\varepsilon_{i}^{n}=\widetilde{u}_{i}^{n}-u_{i}^{n}$, there exists

$$
\begin{aligned}
& \left(1-\kappa_{\Delta x, \tau} g_{i, i}^{\alpha}\right) \varepsilon_{i}^{1}-\kappa_{\Delta x, \tau} \sum_{m=0, m \neq i}^{N_{x}} g_{i, m}^{\alpha} \varepsilon_{m}^{1}=\varepsilon_{i}^{0} e^{-\sigma(n-1) \tau}, \quad n=1, \\
& \left(1-\kappa_{\Delta x, \tau} g_{i, i}^{\alpha}\right) \varepsilon_{i}^{n}-\kappa_{\Delta x, \tau} \sum_{m=0, m \neq i}^{N_{x}} g_{i, m}^{\alpha} \varepsilon_{m}^{n}=\sum_{k=1}^{n-1} \varepsilon_{i}^{k} e^{-\sigma(n-1-k) \tau}\left(1-e^{-\sigma \tau}\right)+\varepsilon_{i}^{0} e^{-\sigma(n-1) \tau}, n>1 .
\end{aligned}
$$

We next prove the scheme is unconditionally stable by the mathematical induction.

Let $E^{n}=\left[\varepsilon_{0}^{n}, \varepsilon_{1}^{n}, \cdots, \varepsilon_{N_{x}}^{n}\right]$ and $\left|\varepsilon_{i_{0}}^{n}\right|:=|| E^{n} \|_{\infty}=\max _{0 \leq i \leq N_{x}}\left|\varepsilon_{i}^{n}\right|$. From Lemma3.3, we obtain

$$
\begin{aligned}
|| E^{1} \|_{\infty} & =\left|\varepsilon_{i_{0}}^{1}\right| \leq\left|\varepsilon_{i_{0}}^{1}\right|-\kappa_{\Delta x, \tau} \sum_{m=0}^{N_{x}} g_{i_{0}, m}^{\alpha}\left|\varepsilon_{i_{0}}^{1}\right|=\left|\varepsilon_{i_{0}}^{1}\right|-\kappa_{\Delta x, \tau} g_{i_{0}, i_{0}}^{\alpha}\left|\varepsilon_{i_{0}}^{1}\right|-\kappa_{\Delta x, \tau} \sum_{m=0, m \neq i_{0}}^{N_{x}} g_{i_{0}, m}^{\alpha}\left|\varepsilon_{i_{0}}^{1}\right| \\
& \leq\left(1-\kappa_{\Delta x, \tau} g_{i_{0}, i_{0}}^{\alpha}\right)\left|\varepsilon_{i_{0}}^{1}\right|-\kappa_{\Delta x, \tau} \sum_{m=0, m \neq i_{0}}^{N_{x}} g_{i_{0}, m}^{\alpha}\left|\varepsilon_{m}^{1}\right| \\
& \leq\left|\left(1-\kappa_{\Delta x, \tau} g_{i_{0}, i_{0}}^{\alpha}\right) \varepsilon_{i_{0}}^{1}-\kappa_{\Delta x, \tau} \sum_{m=0, m \neq i_{0}}^{N_{x}} g_{i_{0}, m}^{\alpha} \varepsilon_{m}^{1}\right|=\left|\varepsilon_{i_{0}}^{0}\right| e^{-\sigma(n-1) \tau} \leq\left\|E^{0}\right\|_{\infty} .
\end{aligned}
$$

Assuming $\left\|E^{s}\right\|_{\infty} \leq\left\|E^{0}\right\|_{\infty}, s=1,2,3, \cdots, n-1$, we have

$$
\begin{aligned}
\left\|E^{n}\right\|_{\infty} & =\left|\varepsilon_{i_{0}}^{n}\right| \leq\left|\varepsilon_{i_{0}}^{n}\right|-\kappa_{\Delta x, \tau} \sum_{m=0}^{N_{x}} g_{i_{0}, m}^{\alpha}\left|\varepsilon_{i_{0}}^{n}\right|=\left|\varepsilon_{i_{0}}^{n}\right|-\kappa_{\Delta x, \tau} g_{i_{0}, i_{0}}^{\alpha}\left|\varepsilon_{i_{0}}^{n}\right|-\kappa_{\Delta x, \tau} \sum_{m=0, m \neq i_{0}}^{N_{x}} g_{i_{0}, m}^{\alpha}\left|\varepsilon_{i_{0}}^{n}\right| \\
& \leq\left(1-\kappa_{\Delta x, \tau} g_{i_{0}, i_{0}}^{\alpha}\right)\left|\varepsilon_{i_{0}}^{n}\right|-\kappa_{\Delta x, \tau} \sum_{m=0, m \neq i_{0}}^{N_{x}} g_{i_{0}, m}^{\alpha}\left|\varepsilon_{m}^{n}\right| \\
& \leq\left|\left(1-\kappa_{\Delta x, \tau} g_{i_{0}, i_{0}}^{\alpha}\right) \varepsilon_{i_{0}}^{n}-\kappa_{\Delta x, \tau} \sum_{m=0, m \neq i_{0}}^{N_{x}} g_{i_{0}, m}^{\alpha} \varepsilon_{m}^{n}\right| \\
& =\left|\sum_{k=1}^{n-1} \varepsilon_{i_{0}}^{k} e^{-\sigma(n-1-k) \tau}\left(1-e^{-\sigma \tau}\right)+\varepsilon_{i_{0}}^{0} e^{-\sigma(n-1) \tau}\right| \\
& \leq\left|\sum_{k=1}^{n-1} e^{-\sigma(n-1-k) \tau}\left(1-e^{-\sigma \tau}\right)+e^{-\sigma(n-1) \tau}\right||| E^{0}\left\|_{\infty}=\right\| E^{0} \|_{\infty} .
\end{aligned}
$$

The proof is completed.

Theorem 3.4 Let $u_{i}^{n}$ be the approximate solution of $u\left(x_{i}, t_{n}\right)$ computed by the difference scheme 2.12. Let $\varepsilon_{i}^{n}=u\left(x_{i}, t_{n}\right)-u_{i}^{n}$. Then

$$
\left\|\varepsilon^{n}\right\|_{\infty} \leq(1-\gamma)\left(\sigma T+e^{\sigma \tau}\right) C_{u}\left(\tau^{2}+(\Delta x)^{2}\right),
$$

where $C_{u}$ is defined by (2.9) and $\left(x_{i}, t_{n}\right) \in(0, b) \times(0, T]$ with $N \tau \leq T$. 
Proof Let $u\left(x_{i}, t_{n}\right)$ be the exact solution of (2.3) at the mesh point $\left(x_{i}, t_{n}\right)$. Defined $\varepsilon_{i}^{n}=$ $u\left(x_{i}, t_{n}\right)-u_{i}^{n}$ and $\varepsilon^{n}=\left[\varepsilon_{0}^{n}, \varepsilon_{1}^{n}, \cdots, \varepsilon_{N_{x}}^{n}\right]$. Subtracting (2.8) from (2.12) with $\varepsilon_{i}^{0}=0$, we obtain

$$
\begin{aligned}
& \left(1-\kappa_{\Delta x, \tau} g_{i, i}^{\alpha}\right) \varepsilon_{i}^{1}-\kappa_{\Delta x, \tau} \sum_{m=0, m \neq i}^{N_{x}} g_{i, m}^{\alpha} \varepsilon_{m}^{1}=\frac{(1-\gamma) \sigma \tau}{1-e^{-\sigma \tau}} r_{i}^{1}, \quad n=1, \\
& \left(1-\kappa_{\Delta x, \tau} g_{i, i}^{\alpha}\right) \varepsilon_{i}^{n}-\kappa_{\Delta x, \tau} \sum_{m=0, m \neq i}^{N_{x}} g_{i, m}^{\alpha} \varepsilon_{m}^{n}=\sum_{k=1}^{n-1} \varepsilon_{i}^{k} e^{-\sigma(n-1-k) \tau}\left(1-e^{-\sigma \tau}\right)+\frac{(1-\gamma) \sigma \tau}{1-e^{-\sigma \tau}} r_{i}^{n}, n>1 .
\end{aligned}
$$

We next prove the desired results by the mathematical induction.

Let $\left|\varepsilon_{i_{0}}^{n}\right|:=|| \varepsilon^{n} \|_{\infty}=\max _{0 \leq i \leq N_{x}}\left|\varepsilon_{i}^{n}\right|$ and $r_{\max }=\max _{0 \leq i \leq N_{x}, 0 \leq n \leq N}\left|r_{i}^{n}\right|$. Using Lemma3.3, we have

$$
\begin{aligned}
\left\|\varepsilon^{1}\right\|_{\infty} & =\left|\varepsilon_{i_{0}}^{1}\right| \leq\left|\varepsilon_{i_{0}}^{1}\right|-\kappa_{\Delta x, \tau} \sum_{m=0}^{N_{x}} g_{i_{0}, m}^{\alpha}\left|\varepsilon_{i_{0}}^{1}\right|=\left|\varepsilon_{i_{0}}^{1}\right|-\kappa_{\Delta x, \tau} g_{i_{0}, i_{0}}^{\alpha}\left|\varepsilon_{i_{0}}^{1}\right|-\kappa_{\Delta x, \tau} \sum_{m=0, m \neq i_{0}}^{N_{x}} g_{i_{0}, m}^{\alpha}\left|\varepsilon_{i_{0}}^{1}\right| \\
& \leq\left(1-\kappa_{\Delta x, \tau} g_{i_{0}, i_{0}}^{\alpha}\right)\left|\varepsilon_{i_{0}}^{1}\right|-\kappa_{\Delta x, \tau} \sum_{m=0, m \neq i_{0}}^{N_{x}} g_{i_{0}, m}^{\alpha}\left|\varepsilon_{m}^{1}\right| \\
& \leq\left|\left(1-\kappa_{\Delta x, \tau} g_{i_{0}, i_{0}}^{\alpha}\right) \varepsilon_{i_{0}}^{1}-\kappa_{\Delta x, \tau} \sum_{m=0, m \neq i_{0}}^{N_{x}} g_{i_{0}, m}^{\alpha} \varepsilon_{m}^{1}\right|=\left|\frac{(1-\gamma) \sigma \tau}{1-e^{-\sigma \tau}} r_{i_{0}}^{1}\right| \leq \frac{(1-\gamma) \sigma \tau}{1-e^{-\sigma \tau}} r_{\max } .
\end{aligned}
$$

Supposing $\left\|e^{n}\right\|_{\infty}=\left|\varepsilon_{i_{0}}^{n}\right|=\max _{0 \leq i \leq N_{x}}\left|\varepsilon_{i}^{n}\right|$ and

$$
\left\|e^{s}\right\|_{\infty} \leq \frac{(s-1)\left(1-e^{-\sigma \tau}\right)+1}{1-e^{-\sigma \tau}}(1-\gamma) \sigma \tau r_{\max }, \quad s=1,2,3, \cdots, n-1,
$$

we have

$$
\begin{aligned}
\|\left.\varepsilon^{n}\right|_{\infty} & =\left|\varepsilon_{i_{0}}^{n}\right| \leq\left|\varepsilon_{i_{0}}^{n}\right|-\kappa_{\Delta x, \tau} \sum_{m=0}^{N_{x}} g_{i_{0}, m}^{\alpha}\left|\varepsilon_{i_{0}}^{n}\right|=\left|\varepsilon_{i_{0}}^{n}\right|-\kappa_{\Delta x, \tau} g_{i_{0}, i_{0}}^{\alpha}\left|\varepsilon_{i_{0}}^{n}\right|-\kappa_{\Delta x, \tau} \sum_{m=0, m \neq i_{0}}^{N_{x}} g_{i_{0}, m}^{\alpha}\left|\varepsilon_{i_{0}}^{n}\right| \\
& \leq\left(1-\kappa_{\Delta x, \tau} g_{i_{0}, i_{0}}^{\alpha}\right)\left|\varepsilon_{i_{0}}^{n}\right|-\kappa_{\Delta x, \tau} \sum_{m=0, m \neq i_{0}}^{N_{x}} g_{i_{0}, m}^{\alpha}\left|\varepsilon_{m}^{n}\right| \\
& \leq\left|\left(1-\kappa_{\Delta x, \tau} g_{i_{0}, i_{0}}^{\alpha}\right) \varepsilon_{i_{0}}^{n}-\kappa_{\Delta x, \tau} \sum_{m=0, m \neq i_{0}}^{N_{x}} g_{i_{0}, m}^{\alpha} \varepsilon_{m}^{n}\right| \\
& =\left|\sum_{k=1}^{n-1} \varepsilon_{i_{0}}^{k} e^{-\sigma(n-1-k) \tau}\left(1-e^{-\sigma \tau}\right)+\frac{(1-\gamma) \sigma \tau}{1-e^{-\sigma \tau}} r_{i_{0}}^{n}\right| \leq \Phi_{\tau} \cdot r_{\max }
\end{aligned}
$$

with

$$
\begin{aligned}
\Phi_{\tau} & =\sum_{k=1}^{n-1} \frac{k-(k-1) e^{-\sigma \tau}}{1-e^{-\sigma \tau}}(1-\gamma) \sigma \tau \cdot e^{-\sigma(n-1-k) \tau}\left(1-e^{-\sigma \tau}\right)+\frac{(1-\gamma) \sigma \tau}{1-e^{-\sigma \tau}} \\
& =\frac{(n-1)\left(1-e^{-\sigma \tau}\right)+1}{1-e^{-\sigma \tau}}(1-\gamma) \sigma \tau<(1-\gamma) \sigma T+e^{\theta \sigma \tau}(1-\gamma),
\end{aligned}
$$

where we use $e^{x}=1+e^{\theta x} x, 0<\theta<1$.

According to 2.9], we can get

$$
\left\|\varepsilon^{n}\right\|_{\infty} \leq(1-\gamma)\left(\sigma T+e^{\theta \sigma \tau}\right) r_{\max } \leq(1-\gamma)\left(\sigma T+e^{\sigma \tau}\right) C_{u}\left(\tau^{2}+(\Delta x)^{2}\right) .
$$

The proof is completed. 
3.3 Convergence and stability for $2 \mathrm{D}$

In this subsection, the stability and convergence are obtained by mathematical induction with $L_{\infty}$ norm.

Theorem 3.5 The difference scheme (2.16) is unconditionally stable.

Proof Let $\widetilde{u}_{i, j}^{n}$ be the approximate solution of $u_{i, j}^{n}$, which is the exact solution of the difference scheme 2.16. Denoting $\varepsilon_{i, j}^{n}=\widetilde{u}_{i, j}^{n}-u_{i, j}^{n}$, there exists

$$
\begin{aligned}
& \left(1-\kappa_{\Delta x, \tau}^{\alpha} g_{i, i}^{\alpha}-\kappa_{\Delta y, \tau}^{\beta} g_{i, i}^{\beta}\right) \varepsilon_{i, j}^{1}-\kappa_{\Delta x, \tau}^{\alpha} \sum_{m=0, m \neq i}^{N_{x}} g_{i, m}^{\alpha} \varepsilon_{m, j}^{1}-\kappa_{\Delta y, \tau}^{\beta} \sum_{m=0, m \neq j}^{N_{y}} g_{j, m}^{\alpha} \varepsilon_{i, m}^{1} \\
& =\varepsilon_{i, j}^{0} e^{-\sigma(n-1) \tau}, \quad n=1, \\
& \left(1-\kappa_{\Delta x, \tau}^{\alpha} g_{i, i}^{\alpha}-\kappa_{\Delta y, \tau}^{\beta} g_{i, i}^{\beta}\right) \varepsilon_{i, j}^{n}-\kappa_{\Delta x, \tau}^{\alpha} \sum_{m=0, m \neq i}^{N_{x}} g_{i, m}^{\alpha} \varepsilon_{m, j}^{n}-\kappa_{\Delta y, \tau}^{\beta} \sum_{m=0, m \neq j}^{N_{y}} g_{j, m}^{\alpha} \varepsilon_{i, m}^{n} \\
& =\sum_{k=1}^{n-1} \varepsilon_{i, j}^{k} e^{-\sigma(n-1-k) \tau}\left(1-e^{-\sigma \tau}\right)+\varepsilon_{i, j}^{0} e^{-\sigma(n-1) \tau}, n>1 .
\end{aligned}
$$

We next prove the scheme is unconditionally stable by the mathematical induction.

Let $\left|\varepsilon_{i_{0}, j_{0}}^{n}\right|:=|| E^{n} \|_{\infty}=\max _{0 \leq i \leq N_{x}, 0 \leq j \leq N_{y}}\left|\varepsilon_{i, j}^{n}\right|$. From Lemma3.3, we obtain

$$
\begin{aligned}
& \left.|| E^{1}\right|_{\infty}=\left|\varepsilon_{i_{0}, j_{0}}^{1}\right| \leq\left|\varepsilon_{i_{0}, j_{0}}^{1}\right|-\kappa_{\Delta x, \tau}^{\alpha} \sum_{m=0}^{N_{x}} g_{i_{0}, m}^{\alpha}\left|\varepsilon_{i_{0}, j_{0}}^{1}\right|-\kappa_{\Delta y, \tau}^{\beta} \sum_{m=0}^{N_{y}} g_{j_{0}, m}^{\beta}\left|\varepsilon_{i_{0}, j_{0}}^{1}\right| \\
& \leq\left(1-\kappa_{\Delta x, \tau}^{\alpha} g_{i_{0}, i_{0}}^{\alpha}-\kappa_{\Delta y, \tau}^{\beta} g_{j_{0}, j_{0}}^{\beta}\right)\left|\varepsilon_{i_{0}}^{1}\right|-\kappa_{\Delta x, \tau}^{\alpha} \sum_{m=0, m \neq i_{0}}^{N_{x}} g_{i_{0}, m}^{\alpha}\left|\varepsilon_{m, j_{0}}^{1}\right|-\kappa_{\Delta y, \tau}^{\beta} \sum_{m=0, m \neq j_{0}}^{N_{y}} g_{j_{0}, m}^{\alpha}\left|\varepsilon_{i_{0}, m}^{1}\right| \\
& \leq\left|\left(1-\kappa_{\Delta x, \tau}^{\alpha} g_{i_{0}, i_{0}}^{\alpha}-\kappa_{\Delta y, \tau}^{\beta} g_{j_{0}, j_{0}}^{\beta}\right) \varepsilon_{i_{0}}^{1}-\kappa_{\Delta x, \tau}^{\alpha} \sum_{m=0, m \neq i_{0}}^{N_{x}} g_{i_{0}, m}^{\alpha} \varepsilon_{m, j_{0}}^{1}-\kappa_{\Delta y, \tau}^{\beta} \sum_{m=0, m \neq j_{0}}^{N_{y}} g_{j_{0}, m}^{\alpha} \varepsilon_{i_{0}, m}^{1}\right| \\
& =\left|\varepsilon_{i_{0}, j_{0}}^{0}\right| e^{-\sigma(n-1) \tau} \leq\left\|E^{0}\right\|_{\infty} .
\end{aligned}
$$

Assuming $\left\|E^{s}\right\|_{\infty} \leq\left\|E^{0}\right\|_{\infty}, s=1,2,3, \cdots, n-1$, we have

$$
\begin{aligned}
& || E^{n} \|_{\infty}=\left|\varepsilon_{i_{0}, j_{0}}^{n}\right| \leq\left|\varepsilon_{i_{0}, j_{0}}^{n}\right|-\kappa_{\Delta x, \tau}^{\alpha} \sum_{m=0}^{N_{x}} g_{i_{0}, m}^{\alpha}\left|\varepsilon_{i_{0}, j_{0}}^{n}\right|-\kappa_{\Delta y, \tau}^{\beta} \sum_{m=0}^{N_{y}} g_{j_{0}, m}^{\beta}\left|\varepsilon_{i_{0}, j_{0}}^{n}\right| \\
& \leq\left(1-\kappa_{\Delta x, \tau}^{\alpha} g_{i_{0}, i_{0}}^{\alpha}-\kappa_{\Delta y, \tau}^{\beta} g_{j_{0}, j_{0}}^{\beta}\right)\left|\varepsilon_{i_{0}}^{n}\right|-\kappa_{\Delta x, \tau}^{\alpha} \sum_{m=0, m \neq i_{0}}^{N_{x}} g_{i_{0}, m}^{\alpha}\left|\varepsilon_{m, j_{0}}^{n}\right|-\kappa_{\Delta y, \tau}^{\beta} \sum_{m=0, m \neq j_{0}}^{N_{y}} g_{j_{0}, m}^{\alpha}\left|\varepsilon_{i_{0}, m}^{n}\right| \\
& \leq\left|\left(1-\kappa_{\Delta x, \tau}^{\alpha} g_{i_{0}, i_{0}}^{\alpha}-\kappa_{\Delta y, \tau}^{\beta} g_{j_{0}, j_{0}}^{\beta}\right) \varepsilon_{i_{0}}^{n}-\kappa_{\Delta x, \tau}^{\alpha} \sum_{m=0, m \neq i_{0}}^{N_{x}} g_{i_{0}, m}^{\alpha} \varepsilon_{m, j_{0}}^{n}-\kappa_{\Delta y, \tau}^{\beta} \sum_{m=0, m \neq j_{0}}^{N_{y}} g_{j_{0}, m}^{\alpha} \varepsilon_{i_{0}, m}^{n}\right| \\
& =\left|\sum_{k=1}^{n-1} \varepsilon_{i_{0}, j_{0}}^{k} e^{-\sigma(n-1-k) \tau}\left(1-e^{-\sigma \tau}\right)+\varepsilon_{i_{0}, j_{0}}^{0} e^{-\sigma(n-1) \tau}\right| \\
& \leq\left|\sum_{k=1}^{n-1} e^{-\sigma(n-1-k) \tau}\left(1-e^{-\sigma \tau}\right)+e^{-\sigma(n-1) \tau}\right||| E^{0}\left\|_{\infty}=|| E^{0}\right\|_{\infty} .
\end{aligned}
$$

The proof is completed. 
Title Suppressed Due to Excessive Length

15

Theorem 3.6 Let $u_{i, j}^{n}$ be the approximate solution of $u\left(x_{i}, y_{j}, t_{n}\right)$ computed by the difference scheme 2.16. Let $\varepsilon_{i, j}^{n}=u\left(x_{i}, y_{j}, t_{n}\right)-u_{i, j}^{n}$. Then

$$
\left\|\varepsilon^{n}\right\|_{\infty} \leq(1-\gamma)\left(\sigma T+e^{\sigma \tau}\right) C_{u}\left(\tau^{2}+(\Delta x)^{2}+(\Delta y)^{2}\right)
$$

where $C_{u}$ is defined by (2.9) and $\left(x_{i}, y_{j}, t_{n}\right) \in \Omega \times(0, T]$ with $N \tau \leq T$.

Proof Let $u\left(x_{i}, y_{j}, t_{n}\right)$ be the exact solution of 1.1) at the mesh point $\left(x_{i}, y_{j}, t_{n}\right)$. Defined $\varepsilon_{i, j}^{n}=u\left(x_{i}, y_{j}, t_{n}\right)-u_{i, j}^{n}$. Subtracting (2.13) from 2.16) with $\varepsilon_{i, j}^{0}=0$, we obtain

$$
\begin{aligned}
& \left(1-\kappa_{\Delta x, \tau}^{\alpha} g_{i, i}^{\alpha}-\kappa_{\Delta y, \tau}^{\beta} g_{i, i}^{\beta}\right) \varepsilon_{i, j}^{1}-\kappa_{\Delta x, \tau}^{\alpha} \sum_{m=0, m \neq i}^{N_{x}} g_{i, m}^{\alpha} \varepsilon_{m, j}^{1}-\kappa_{\Delta y, \tau}^{\beta} \sum_{m=0, m \neq j}^{N_{y}} g_{j, m}^{\alpha} \varepsilon_{i, m}^{1} \\
& =\frac{(1-\gamma) \sigma \tau}{1-e^{-\sigma \tau}} r_{i, j}^{1}, \quad n=1, \\
& \left(1-\kappa_{\Delta x, \tau}^{\alpha} g_{i, i}^{\alpha}-\kappa_{\Delta y, \tau}^{\beta} g_{i, i}^{\beta}\right) \varepsilon_{i, j}^{n}-\kappa_{\Delta x, \tau}^{\alpha} \sum_{m=0, m \neq i}^{N_{x}} g_{i, m}^{\alpha} \varepsilon_{m, j}^{n}-\kappa_{\Delta y, \tau}^{\beta} \sum_{m=0, m \neq j}^{N_{y}} g_{j, m}^{\alpha} \varepsilon_{i, m}^{n} \\
& =\sum_{k=1}^{n-1} \varepsilon_{i, j}^{k} e^{-\sigma(n-1-k) \tau}\left(1-e^{-\sigma \tau}\right)+\frac{(1-\gamma) \sigma \tau}{1-e^{-\sigma \tau}} r_{i, j}^{n}, n>1 .
\end{aligned}
$$

We next prove the desired results by the mathematical induction.

Let $\left|\varepsilon_{i_{0}, j_{0}}^{n}\right|:=|| \varepsilon^{n} \|_{\infty}=\max _{0 \leq i \leq N_{x}, 0 \leq j \leq N_{y}}\left|\varepsilon_{i, j}^{n}\right|$ and $r_{\max }=\max _{0 \leq i \leq N_{x}, 0 \leq j \leq N_{y}, 0 \leq n \leq N}\left|r_{i, j}^{n}\right|$. Using Lemma 3.3 we have

$$
\begin{aligned}
\mid & \left.\left|\varepsilon^{1}\right|\right|_{\infty}=\left|\varepsilon_{i_{0}, j_{0}}^{1}\right| \leq\left|\varepsilon_{i_{0}, j_{0}}^{1}\right|-\kappa_{\Delta x, \tau}^{\alpha} \sum_{m=0}^{N_{x}} g_{i_{0}, m}^{\alpha}\left|\varepsilon_{i_{0}, j_{0}}^{1}\right|-\kappa_{\Delta y, \tau}^{\beta} \sum_{m=0}^{N_{y}} g_{j_{0}, m}^{\beta}\left|\varepsilon_{i_{0}, j_{0}}^{1}\right| \\
= & \left|\varepsilon_{i_{0}, j_{0}}^{1}\right|-\kappa_{\Delta x, \tau}^{\alpha} g_{i_{0}, i_{0}}^{\alpha}\left|\varepsilon_{i_{0}, j_{0}}^{1}\right|-\kappa_{\Delta x, \tau}^{\alpha} \sum_{m=0, m \neq i_{0}}^{N_{x}} g_{i_{0}, m}^{\alpha}\left|\varepsilon_{i_{0}, j_{0}}^{1}\right| \\
& -\kappa_{\Delta y, \tau}^{\beta} g_{j_{0}, j_{0}}^{\beta}\left|\varepsilon_{i_{0}, j_{0}}^{1}\right|-\kappa_{\Delta y, \tau}^{\beta} \sum_{m=0, m \neq j_{0}}^{N_{y}} g_{j_{0}, m}^{\alpha}\left|\varepsilon_{i_{0}, j_{0}}^{1}\right| \\
\leq & \left(1-\kappa_{\Delta x, \tau}^{\alpha} g_{i_{0}, i_{0}}^{\alpha}-\kappa_{\Delta y, \tau}^{\beta} g_{j_{0}, j_{0}}^{\beta}\right)\left|\varepsilon_{i_{0}, j_{0}}^{1}\right|-\kappa_{\Delta x, \tau}^{\alpha} \sum_{m=0, m \neq i_{0}}^{N_{x}} g_{i_{0}, m}^{\alpha}\left|\varepsilon_{m, j_{0}}^{1}\right|-\kappa_{\Delta y, \tau}^{\beta} \sum_{m=0, m \neq j_{0}}^{N_{y}} g_{j_{0}, m}^{\alpha}\left|\varepsilon_{i_{0}, m}^{1}\right| \\
\leq & \left|\left(1-\kappa_{\Delta x, \tau}^{\alpha} g_{i_{0}, i_{0}}^{\alpha}-\kappa_{\Delta y, \tau}^{\beta} g_{j_{0}, j_{0}}^{\beta}\right) \varepsilon_{i_{0}, j_{0}}^{1}-\kappa_{\Delta x, \tau}^{\alpha} \sum_{m=0, m \neq i_{0}}^{N_{x}} g_{i_{0}, m}^{\alpha} \varepsilon_{m, j_{0}}^{1}-\kappa_{\Delta y, \tau}^{\beta} \sum_{m=0, m \neq j_{0}}^{N_{y}} g_{j_{0}, m}^{\alpha} \varepsilon_{i_{0}, m}^{1}\right| \\
= & \left|\frac{(1-\gamma) \sigma \tau}{1-e^{-\sigma \tau}} r_{i_{0}, j_{0}}^{1}\right| \leq \frac{(1-\gamma) \sigma \tau}{1-e^{-\sigma \tau}} r_{\max } .
\end{aligned}
$$

Supposing $\left.|| \varepsilon^{n}\right|_{\infty}=\left|\varepsilon_{i_{0}, j_{0}}^{n}\right|=\max _{0 \leq i \leq N_{x}, 0 \leq j \leq N_{y}}\left|\varepsilon_{i, j}^{n}\right|$ and

$$
\left\|\varepsilon^{s}\right\|_{\infty} \leq \frac{(s-1)\left(1-e^{-\sigma \tau}\right)+1}{1-e^{-\sigma \tau}}(1-\gamma) \sigma \tau r_{\max }, \quad s=1,2,3, \cdots, n-1,
$$


we have

$$
\begin{aligned}
& || \varepsilon^{n}||_{\infty}=\left|\varepsilon_{i_{0}, j_{0}}^{n}\right| \leq\left|\varepsilon_{i_{0}, j_{0}}^{n}\right|-\kappa_{\Delta x, \tau}^{\alpha} \sum_{m=0}^{N_{x}} g_{i_{0}, m}^{\alpha}\left|\varepsilon_{i_{0}, j_{0}}^{n}\right|-\kappa_{\Delta y, \tau}^{\beta} \sum_{m=0}^{N_{y}} g_{j_{0}, m}^{\beta}\left|\varepsilon_{i_{0}, j_{0}}^{n}\right| \\
= & \left|\varepsilon_{i_{0}, j_{0}}^{n}\right|-\kappa_{\Delta x, \tau}^{\alpha} g_{i_{0}, i_{0}}^{\alpha}\left|\varepsilon_{i_{0}, j_{0}}^{n}\right|-\kappa_{\Delta x, \tau}^{\alpha} \sum_{m=0, m \neq i_{0}}^{N_{x}} g_{i_{0}, m}^{\alpha}\left|\varepsilon_{i_{0}, j_{0}}^{n}\right| \\
& -\kappa_{\Delta y, \tau}^{\beta} g_{j_{0}, j_{0}}^{\beta}\left|\varepsilon_{i_{0}, j_{0}}^{n}\right|-\kappa_{\Delta y, \tau}^{\beta} \sum_{m=0, m \neq j_{0}}^{N_{y}} g_{j_{0}, m}^{\alpha}\left|\varepsilon_{i_{0}, j_{0}}^{n}\right| \\
\leq & \left(1-\kappa_{\Delta x, \tau}^{\alpha} g_{i_{0}, i_{0}}^{\alpha}-\kappa_{\Delta y, \tau}^{\beta} g_{j_{0}, j_{0}}^{\beta}\right)\left|\varepsilon_{i_{0}, j_{0}}^{n}\right|-\kappa_{\Delta x, \tau}^{\alpha} \sum_{m=0, m \neq i_{0}}^{N_{x}} g_{i_{0}, m}^{\alpha}\left|\varepsilon_{m, j_{0}}^{n}\right|-\kappa_{\Delta y, \tau}^{\beta} \sum_{m=0, m \neq j_{0}}^{N_{y}} g_{j_{0}, m}^{\alpha}\left|\varepsilon_{i_{0}, m}^{n}\right| \\
\leq & \left|\left(1-\kappa_{\Delta x, \tau}^{\alpha} g_{i_{0}, i_{0}}^{\alpha}-\kappa_{\Delta y, \tau}^{\beta} g_{j_{0}, j_{0}}^{\beta}\right) \varepsilon_{i_{0}, j_{0}}^{n}-\kappa_{\Delta x, \tau}^{\alpha} \sum_{m=0, m \neq i_{0}}^{N_{x}} g_{i_{0}, m}^{\alpha} \varepsilon_{m, j_{0}}^{n}-\kappa_{\Delta y, \tau}^{\beta} \sum_{m=0, m \neq j_{0}}^{N_{y}} g_{j_{0}, m}^{\alpha} \varepsilon_{i_{0}, m}^{n}\right| \\
= & \left|\sum_{k=1}^{n-1} \varepsilon_{i_{0}, j_{0}}^{k} e^{-\sigma(n-1-k) \tau}\left(1-e^{-\sigma \tau}\right)+\varepsilon_{i_{0}, j_{0}}^{0} e^{-\sigma(n-1) \tau}\right| \leq \Phi_{\tau} \cdot r_{\max },
\end{aligned}
$$

where $\Phi_{\tau}$ is given by (3.4).

According to 2.14, we can get

$$
\left\|\varepsilon^{n}\right\|_{\infty} \leq(1-\gamma)\left(\sigma T+e^{\theta \sigma \tau}\right) r_{\max } \leq(1-\gamma)\left(\sigma T+e^{\sigma \tau}\right) C_{u}\left(\tau^{2}+(\Delta x)^{2}+(\Delta y)^{2}\right) .
$$

The proof is completed.

\section{Numerical results}

In the section, we numerically verify the above theoretical results including convergence orders and numerical stability. And the $l_{\infty}$ norm and the discrete $L^{2}$-norm, respectively, are used to measure the numerical errors.

\section{Example 1}

Consider 2.3) on a finite domain with $0<x<1,0<t \leq 1$, and the forcing function is

$$
\begin{aligned}
f(x, t)= & -\frac{\sigma}{1-\gamma} t e^{-\sigma t} x^{2}(1-x)^{2}+\frac{e^{-\sigma t}}{2 \cos (\alpha \pi / 2)} \frac{24}{\Gamma(5-\alpha)}\left(x^{4-\alpha}+(1-x)^{4-\alpha}\right) \\
& -\frac{e^{-\sigma t}}{2 \cos (\alpha \pi / 2)} \frac{12}{\Gamma(4-\alpha)}\left(x^{3-\alpha}+(1-x)^{3-\alpha}\right) \\
& +\frac{e^{-\sigma t}}{2 \cos (\alpha \pi / 2)} \frac{2}{\Gamma(3-\alpha)}\left(x^{2-\alpha}+(1-x)^{2-\alpha}\right), \quad \sigma=\frac{\gamma}{1-\gamma}
\end{aligned}
$$

with the nonzero initial condition $u(x, 0)=x^{2}(1-x)^{2}$ and the homogeneous Dirichlet boundary conditions. The exact solution of the fractional PDEs is

$$
u(x, t)=e^{-\sigma t} x^{2}(1-x)^{2} .
$$

Tables 1 and 2 show that the schemes 2.11) have the global truncation errors $\mathscr{O}\left(\tau^{2}+\right.$ $\left.(\Delta x)^{2}\right)$ at time $T=1$. Here the $l_{\infty}$ norm and the discrete $L^{2}$-norm, respectively, are used to measure the numerical errors for 2.11 with $\tau=\Delta x$. 
Table 1 The maximum errors and convergence orders for 2.11 with $\tau=\Delta x$.

\begin{tabular}{lccccc}
\hline$l_{\infty}$ norm & $\tau$ & $\alpha=1.2$ & Rate & $\alpha=1.8$ & Rate \\
\hline \multirow{3}{*}{$\gamma=0.1$} & $1 / 40$ & $1.0686 \mathrm{e}-04$ & & $1.3426 \mathrm{e}-04$ & \\
& $1 / 80$ & $2.9917 \mathrm{e}-05$ & 1.8367 & $3.3543 \mathrm{e}-05$ & 2.0009 \\
& $1 / 160$ & $7.9022 \mathrm{e}-06$ & 1.9206 & $8.3559 \mathrm{e}-06$ & 2.0051 \\
& $1 / 320$ & $2.0766 \mathrm{e}-06$ & 1.9281 & $2.0766 \mathrm{e}-06$ & 2.0086 \\
\hline \multirow{3}{*}{$\gamma=0.5$} & $1 / 40$ & $4.4671 \mathrm{e}-05$ & & $6.0820 \mathrm{e}-05$ & \\
& $1 / 80$ & $1.2415 \mathrm{e}-05$ & 1.8473 & $1.5196 \mathrm{e}-05$ & 2.0009 \\
& $1 / 160$ & $3.2730 \mathrm{e}-06$ & 1.9234 & $3.7834 \mathrm{e}-06$ & 2.0059 \\
& $1 / 320$ & $8.6348 \mathrm{e}-07$ & 1.9224 & $9.3952 \mathrm{e}-07$ & 2.0097 \\
\hline \multirow{3}{*}{$\gamma=0.9$} & $1 / 40$ & $2.9977 \mathrm{e}-05$ & & $6.8820 \mathrm{e}-06$ & \\
& $1 / 80$ & $7.4790 \mathrm{e}-06$ & 2.0029 & $1.7237 \mathrm{e}-06$ & 1.9973 \\
& $1 / 160$ & $1.8634 \mathrm{e}-06$ & 2.0049 & $4.3057 \mathrm{e}-07$ & 2.0012 \\
& $1 / 320$ & $4.6419 \mathrm{e}-07$ & 2.0051 & $1.0735 \mathrm{e}-07$ & 2.0040 \\
\hline
\end{tabular}

Table 2 The discrete $L^{2}$-norm errors and convergence orders for 2.11 with $\tau=\Delta x$.

\begin{tabular}{lccccc}
\hline$L^{2}$-norm & $\tau$ & $\alpha=1.2$ & Rate & $\alpha=1.8$ & Rate \\
\hline \multirow{3}{*}{$\gamma=0.1$} & $1 / 40$ & $6.6304 \mathrm{e}-05$ & & $8.9805 \mathrm{e}-05$ & \\
& $1 / 80$ & $1.6925 \mathrm{e}-05$ & 1.9700 & $2.2274 \mathrm{e}-05$ & 2.0114 \\
& $1 / 160$ & $4.3290 \mathrm{e}-06$ & 1.9670 & $5.5195 \mathrm{e}-06$ & 2.0128 \\
& $1 / 320$ & $1.1060 \mathrm{e}-06$ & 1.9687 & $1.3670 \mathrm{e}-06$ & 2.0135 \\
\hline \multirow{3}{*}{$\gamma=0.5$} & $1 / 40$ & $3.0386 \mathrm{e}-05$ & & $4.0823 \mathrm{e}-05$ & \\
& $1 / 80$ & $7.6617 \mathrm{e}-06$ & 1.9877 & $1.0123 \mathrm{e}-05$ & 2.0118 \\
& $1 / 160$ & $1.9408 \mathrm{e}-06$ & 1.9810 & $2.5056 \mathrm{e}-06$ & 2.0144 \\
& $1 / 320$ & $4.9221 \mathrm{e}-07$ & 1.9793 & $6.1953 \mathrm{e}-07$ & 2.0159 \\
\hline \multirow{3}{*}{$\begin{array}{c}* \\
\gamma\end{array}$} & $1 / 40$ & $2.2186 \mathrm{e}-05$ & & $4.9221 \mathrm{e}-06$ & \\
& $1 / 80$ & $5.5359 \mathrm{e}-06$ & 2.0027 & $1.2327 \mathrm{e}-06$ & 1.9975 \\
& $1 / 160$ & $1.3791 \mathrm{e}-06$ & 2.0050 & $3.0788 \mathrm{e}-07$ & 2.0014 \\
& $1 / 320$ & $3.4350 \mathrm{e}-07$ & 2.0054 & $7.6749 \mathrm{e}-08$ & 2.0042 \\
\hline
\end{tabular}

\section{Example 2}

Consider (1.1) on a finite domain with $0<x<1,0<y<1,0<t \leq 1$, and the forcing function is

$$
\begin{aligned}
f(x, y, t)= & -\frac{\sigma}{1-\gamma} t e^{-\sigma t} x^{2}(1-x)^{2} y^{2}(1-y)^{2}+\frac{e^{-\sigma t} y^{2}(1-y)^{2}}{2 \cos (\alpha \pi / 2)} \frac{24\left(x^{4-\alpha}+(1-x)^{4-\alpha}\right)}{\Gamma(5-\alpha)} \\
& +\frac{e^{-\sigma t}}{2 \cos (\alpha \pi / 2)} y^{2}(1-y)^{2}\left(\frac{2\left(x^{2-\alpha}+(1-x)^{2-\alpha}\right)}{\Gamma(3-\alpha)}-\frac{12\left(x^{3-\alpha}+(1-x)^{3-\alpha}\right)}{\Gamma(4-\alpha)}\right) \\
& +\frac{e^{-\sigma t}}{2 \cos (\alpha \pi / 2)} x^{2}(1-x)^{2}\left(\frac{24\left(y^{4-\alpha}+(1-y)^{4-\alpha}\right)}{\Gamma(5-\alpha)}-\frac{12\left(y^{3-\alpha}+(1-y)^{3-\alpha}\right)}{\Gamma(4-\alpha)}\right) \\
& +\frac{e^{-\sigma t}}{2 \cos (\alpha \pi / 2)} x^{2}(1-x)^{2} \frac{2}{\Gamma(3-\alpha)}\left(y^{2-\alpha}+(1-y)^{2-\alpha}\right), \quad \sigma=\frac{\gamma}{1-\gamma}
\end{aligned}
$$


with the nonzero initial condition $u(x, y, 0)=x^{2}(1-x)^{2} y^{2}(1-y)^{2}$ and the homogeneous Dirichlet boundary conditions. The exact solution of the (1.1) is

$$
u(x, y, t)=e^{-\sigma t} x^{2}(1-x)^{2} y^{2}(1-y)^{2} .
$$

Table 3 The maximum errors and convergence orders for 2.16 with $\tau=\Delta x=\Delta y$.

\begin{tabular}{lccccc}
\hline$l_{\infty}$ norm & $\tau$ & $\alpha=1.2, \beta=1.3$ & Rate & $\alpha=1.8, \beta=1.7$ & Rate \\
\hline \multirow{3}{*}{$\gamma=0.3$} & $1 / 10$ & $8.7959 \mathrm{e}-05$ & & $1.0126 \mathrm{e}-04$ & \\
& $1 / 20$ & $2.1543 \mathrm{e}-05$ & 2.0296 & $2.5618 \mathrm{e}-05$ & 1.9828 \\
& $1 / 40$ & $5.2815 \mathrm{e}-06$ & 2.0282 & $6.4708 \mathrm{e}-06$ & 1.9851 \\
& $1 / 80$ & $1.3016 \mathrm{e}-06$ & 2.0207 & $1.6713 \mathrm{e}-06$ & 1.9530 \\
\hline \multirow{3}{*}{$\gamma=0.7$} & $1 / 10$ & $2.2733 \mathrm{e}-05$ & & $1.8114 \mathrm{e}-05$ & \\
& $1 / 20$ & $5.5809 \mathrm{e}-06$ & 2.0262 & $4.5869 \mathrm{e}-06$ & 1.9815 \\
& $1 / 40$ & $1.3680 \mathrm{e}-06$ & 2.0284 & $1.1568 \mathrm{e}-06$ & 1.9873 \\
& $1 / 80$ & $3.4094 \mathrm{e}-07$ & 2.0045 & $2.9480 \mathrm{e}-07$ & 1.9723 \\
\hline
\end{tabular}

Tables 3 shows that the maximum error, at time $T=1$ and $\tau=\Delta x=\Delta y$, between the exact analytical value and the numerical value. The scheme 2.16) is second-order convergence and this is in agreement with the order of the truncation error.

\section{Conclusions}

As is well known, there is less than the second-order convergence for the Caputo fractional derivative [18,23] with L1 formula. We notice that there are already some theoretical convergence results for Caputo-Fabrizio fractional derivative [3.7] with L1 formula. However, it seems that achieving a second-order accurate scheme (optimal estimates) is not an easy task. To our knowledge, this is the first published finite difference method to consider the space fractional diffusion equations with the time Caputo-Fabrizio fractional derivative. The optimal estimates with the second-order convergence for L1 scheme are given by two methods. We remark that the corresponding theoretical including a prior estimate can also be extended to the nonzero initial values [13]17].

\section{Acknowledgements}

This work was supported by NSFC 11601206.

\section{References}

1. Abdulhameed, M., Muhammad, M.M., Gital, A.Y., Yakubu, D.G., Khan, I.: Effect of fractional derivatives on transient MHD flow and radiative heat transfer in a micro-parallel channel at high zeta potentials. Phys. A. 484, 42-71 (2019).

2. Abdulhameed, M., Vieru, D., Roslan, R.: Magnetohydrodynamic electroosmotic flow of Maxwell fluids with Caputo-Fabrizio derivatives through circular tubes. Comput. Math. Appl. 74, 2503-2519 (2017). 
3. Akman, T., Yildiz, B., Baleanu, D.: New discretization of Caputo-Fabrizio derivative. Comp. Appl. Math. 37, 3307-3333 (2018).

4. Alkahtani, B.S.T., Atangana, A.: Controlling the wave movement on the surface of shallow water with the Caputo-Fabrizio derivative with fractional order. Chaos. Soliton. Fract. 89, 539-546 (2016).

5. Asif, N.A., Hammouch, Z., Riaz, M.B., Bulut, H.: Analytical solution of a Maxwell fluid with slip effects in view of the Caputo-Fabrizio derivative. Eur. Phys. J. Plus 133:272 (2018)

6. Atanackovié, T.M., Pilipovié, S., Zorica, D.: Properties of the Caputo-Fabrizio fractional derivative and its distributional settings. Fract. Calc. Appl. Anal. 21, 29-44 (2018).

7. Atangana, A., Alqahtani, R.T.: Numerical approximation of the space-time Caputo-Fabrizio fractional derivative and application to groundwater pollution equation. Adv. Differ. Equ. 2016, 1-13 (2016).

8. Atangana, A., Alkahtani, B.S.T.: New model of groundwater flowing within a confine aquifer: application of Caputo-Fabrizio derivative. Arab. J. Geosci. 9, 1-6 (2016).

9. Caputo,M., Fabrizio, M.: A new definition of fractional derivative without singular kernel. Progr. Fract. Differ. Appl. 1, 73-85 (2015).

10. Chen, M.H., Deng, W.H.: Fourth order accurate scheme for the space fractional diffusion equations. SIAM J. Numer. Anal. 52, 1418-1438 (2014).

11. Chen, M.H., Deng, W.H.: Discretized fractional substantial calculus. ESAIM: M2AN. 49, 373-394 (2015).

12. Chen, M.H., Deng, W.H.: A second-order numerical method for two-dimensional two-sided space fractional convection diffusion equation. Appl. Math. Model. 38 3244-3259 (2014).

13. Chen, M.H., Shi,J.K., Deng, W.H.: High order algorithms for Fokker-Planck equation with CaputoFabrizio fractional derivative. arXiv:1809.03263

14. Chen, M.H., Wang, Y.T., Cheng, X., Deng, W.H.: Second-order LOD multigrid method for multidimensional Riesz fractional diffusion equation. BIT. 54 623-647 (2014).

15. Djida, J.D., Atangana, A.: More generalized groundwater model with space-time Caputo Fabrizio fractional differentiation. Numer. Meth. Part. D. E. 33 1616-1627 (2017).

16. Firoozjaee, M.A., Jafari, H., Lia, A., Baleanu, D.: Numerical approach of Fokker-Planck equation with Caputo-Fabrizio fractional derivative using Ritz approximation. J. Comput. Appl. Math. 339 367-373 (2018).

17. Ji, C.C., Sun, Z.Z.: A high-order compact finite difference schemes for the fractional sub-diffusion equation. J. Sci. Comput. 64, 959-985 (2015).

18. Lin, Y.M., Xu, C.J.: Finite difference/spectral approximations for the time-fractional diffusion equation. J. Comput. Phys. 225, 1533-1552 (2007).

19. Liu, Z.G., Cheng, A.J., Li, X.L.: A second order Crank-Nicolson scheme for fractional Cattaneo equation based on new fractional derivative. Appl. Math. Comput. 311, 361-374 (2017)

20. Loh,J.R., Jafari, H., Isah, A., Phang, C., Toh, Y.T.: On the new properties of Caputo-Fabrizio operator and its application in deriving shifted Lagendre operational matrix. Appl. Numer. Math. 132, 138-153 (2018).

21. Lubich, Ch.: Discretized fractional calculus. SIAM J. Math. Anal. 17, 704-719 (1986).

22. Mahsud, Y., Shah, N.A., Vieru, D.: Natural convection flows and heat transfer with exponential memory of a Maxwell fluid with damped shear stress. Comput. Math. Appl. 76, 2246-2261 (2018).

23. Oldham, K., Spanier, J.: The Fractional Calculus: Theory and Applications of Differentiation and Integration to Arbitrary Order. Academic Press, (1974).

24. Podlubny, I.: Fractional Differential Equations. Academic Press, (1999).

25. Quarteroni, A., Sacco, R., Saleri, F.: Numerical Mathematics. Springer, (2007).

26. Shah, N.A., Khan, I.: Heat transfer analysis in a second grade fluid over and oscillating vertical plate using fractional Caputo-Fabrizio derivatives. Eur. Phys. J. C 76:362 (2016).

27. Ullan, S., Khan, M.A., Farooq, M.: A new fractional model for the dynamics of the hepatitis B virus using the Caputo-Fabrizio derivative. Eur. Phys. J. Plus 133:237 (2018).

28. Zhao, X., Sun, Z.Z.: Compact Crank-Nicolson schemes for a class of fractional Cattaneo equation in inhomogeneous medium. J. Sci. Comput. 62, 747-771 (2014). 